Check for updates

Cite this: RSC Adv., 2017, 7, 55555

Received 25th September 2017 Accepted 24th November 2017 DOI: $10.1039 / c 7 r a 10626 b$

rsc.li/rsc-advances

\section{Nitrogen doped nanoporous graphene: an efficient metal-free electrocatalyst for the oxygen reduction reaction $\uparrow$}

Amir Yadegari, ${ }^{\text {ab }}$ Leila Samiee, ${ }^{c}$ Saeedeh Tasharrofi, ${ }^{\mathrm{c}}$ Sanaz Tajik, ${ }^{\mathrm{d}}$ Alimorad Rashidi, ${ }^{\mathrm{c}}$

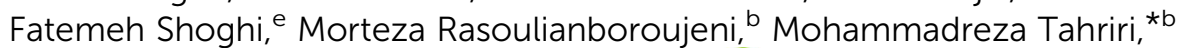

Samuel J. Rowley-Neale ${ }^{f g}$ and Craig E. Banks (D)*fg

The oxygen reduction reaction (ORR) is an important half reaction, which occurs at the cathode within a fuel cell and limits their range of applications due to slow electrochemical kinetics. To overcome this issue, electrocatalysts are sought, which need to be an alternative to expensive and unsustainable metallic catalysts. Herein we report the synthesis of nitrogen doped nanoporous graphene (NPG), which is a competitive alternative to currently employed metallic catalysts. The NPG is synthesised through a chemical vapour deposition methodology followed by a chemical functionalization step introducing oxygen functional groups (carbonyl and hydroxyl groups), which is then doped with nitrogen via orthophenylenediamine (OPDA). The NPG is physiochemically and electrochemically characterised. The NPG demonstrates outstanding electrocatalytic activity towards the ORR in alkaline media proceeding via a favourable 4-electron pathway and is comparable to commercially available platinum-carbon (20\%). We demonstrate that the electrochemical activity of the NPG is tailorable such that through increased nitrogen doping the ORR transforms from a 2-electron process to that of the favourable 4-electron process via increasing the proportion of pyridinic nitrogen while the content of graphitic nitrogen remains almost constant. The NPG exhibits excellent electrochemical performance towards the ORR in alkaline media, long-term stability and appropriate methanol crossover as benchmarked to commercialised Pt/C electrodes; this outstanding electrocatalytic activity is related to the high proportion of defects, high porosity and (pyridinic) doping.

\title{
Introduction
}

${ }^{a}$ School of Chemical Engineering, College of Engineering, University of Tehran, P.O. Box: 11155-4563, Tehran, Iran

${ }^{b}$ Department of Developmental Sciences, Marquette University, Milwaukee, WI 53233, USA. E-mail: mohammadreza.tahriri@marquette.edu

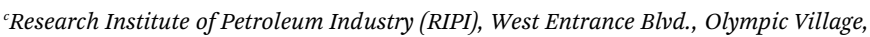
P.O. Box: 14857-33111, Tehran, Iran

${ }^{d}$ Faculty of Chemical Engineering, Amirkabir University of Technology, Hafez Ave, P.O. Box 15875-4413, Tehran, Iran

${ }^{e}$ Department of Chemistry, Université de Montréal, 2900 Boulevard EdouardMontpetit, Montréal, QC H3T 1J4, Canada

${ }^{f}$ Faculty of Science and Engineering, Manchester Metropolitan University, Manchester, M1 5GD, UK. E-mail: c.banks@mmu.ac.uk; Web: http://www.craigbanksresearch.com; Fax: +44 (0)1612476831; Tel: +44 (o)1612471196

${ }^{g}$ Manchester Fuel Cell Innovation Centre, Manchester Metropolitan University, Chester Street, Manchester M1 5GD, UK

$\uparrow$ Electronic supplementary information (ESI) available: BET isotherms of nanoporous and NPG, XPS spectra of wide survey of electrocatalysts and C1s and N1s high resolution spectra, SEM images and mapping analysis of nanoporous graphene and NPG, cyclic voltammetry in $\mathrm{O}_{2}$ or $\mathrm{N}_{2}$ saturated electrolyte and after addition of methanol, chronoamperometry in $\mathrm{O}_{2}$ saturated and after addition of methanol, linear sweep voltammetry at various rotation speeds, Koutecky-Levich plots, and electrochemical impedance spectroscopy of the electrocatalysts. See DOI: 10.1039/c7ra10626b
As the global energy economy reacts to the negative effects of anthropogenic climate change, more sustainable energy systems are being developed. A promising clean energy generation technology involves the use of hydrogen as a fuel source for proton exchange membrane fuel cells (PEMFC), which subsequently generate electrical currents. The key reactions that allow a PEMFC to produce a current are the hydrogen oxidation (HOR) and oxygen reduction reactions (ORR), which occur at the anode and cathode, respectively. Of these two reactions it is the ORR that is the most studied, as it has the most significant influence upon the performance and efficiency of PEMFCs. ${ }^{1-3}$ This is due to the ORR having a large kinetic inhibition, arising due to the strength of the (di)oxygen double bond. ${ }^{4,5}$ The reaction mechanism for the ORR is different for acidic and basic electrolytes as described below and generally an efficient catalyst operates via the 4 electron pathway:

Acidic media:

$$
\mathrm{O}_{2}+4 \mathrm{H}^{+}+4 \mathrm{e}^{-} \rightarrow 2 \mathrm{H}_{2} \mathrm{O}, 4 \text { electron pathway }
$$




$$
\begin{gathered}
\mathrm{O}_{2}+2 \mathrm{H}^{+}+2 \mathrm{e}^{-} \rightarrow \mathrm{H}_{2} \mathrm{O}_{2}, 2 \text { electron pathway } \\
\mathrm{H}_{2} \mathrm{O}_{2}+2 \mathrm{H}^{+}+2 \mathrm{e}^{-} \rightarrow 2 \mathrm{H}_{2} \mathrm{O}
\end{gathered}
$$

Alkaline media:

$$
\begin{gathered}
\mathrm{O}_{2}+2 \mathrm{H}_{2} \mathrm{O}+4 \mathrm{e}^{-} \rightarrow 4 \mathrm{OH}^{-}, 4 \text { electron pathway } \\
\mathrm{O}_{2}+\mathrm{H}_{2} \mathrm{O}+2 \mathrm{e}^{-} \rightarrow \mathrm{HO}_{2}^{-}+\mathrm{OH}^{-}, 2 \text { electron pathway } \\
\mathrm{HO}_{2}^{-}+\mathrm{H}_{2} \mathrm{O}+2 \mathrm{e}^{-} \rightarrow 3 \mathrm{OH}^{-}
\end{gathered}
$$

Attempts to increase the performance of PEMFCs have therefore focused on developing efficient, economical and sustainable new materials to reduce the over-potentials, increase the achievable current density and allow for a 4-electron pathway associated with the ORR. Platinum (Pt), and compounds containing it, are considered the most effective catalysts for ORR allowing the reaction mechanism to occur via a 4-electron pathway. ${ }^{6,7}$ This is a consequence of its low (near negligible) binding energy of adsorbates like $\mathrm{O}_{2}$ and $\mathrm{H}^{+}$. However, the high cost and poor global distribution of platinum limit the attractiveness of its application. ${ }^{8}$

Attempts have been made to develop non-precious metal catalysts as cost effective alternatives to Pt. Recently, carbon nanostructures, such as carbon nanotubes, ${ }^{\mathbf{9 , 1 0}}$ graphene $\mathrm{e}^{\mathbf{1 1 - 1 3}}$ carbon quantum dots, ${ }^{\mathbf{1 4}}$ and mesoporous carbon, ${ }^{15}$ which have been doped with heteroatoms (N, S, P, B, I, or F), have shown promising metal free ORR catalysis. ${ }^{16}$ Table 1 presents a thorough overview of the surrounding literature. The introduction of heteroatoms increases the electron density near the Fermi level, which induces an increase in the electropositive charge on the adjacent carbon atom, via a polarization effect, thus facilitating the binding of the electronegative $\mathrm{O}$ atoms within the electrolyte and making the electropositive carbon atoms the sites responsible for the ORR electrocatalytic activity. ${ }^{17,18}$ Of particular interest within this field has been the utilisation graphene as a carbon material for doping, due to it processing unique and beneficial electrochemical properties compared to other carbon-based materials. ${ }^{19-21}$ whilst, pristine (defect free) graphene possesses poor ORR activity it has been shown that by synthesising mesoporous graphene one can greatly increase graphene's ORR activity. There are several reported methods within the literature for the fabrication of mesoporous graphene, such as; chemical etching, ${ }^{22,23}$ photocatalytic reaction, ${ }^{24}$ lithography, ${ }^{25}$ electron beam ${ }^{26}$ and chemical activation. ${ }^{27}$ An elegant study by Lin et $a l^{28}$ prepared mesoporous nitrogen doped graphene, through pyrolysis of graphene oxide and polyaniline at $1000{ }^{\circ} \mathrm{C}$, and observed it to have beneficial ORR electrocatalytic activity and lowered the electronegativity of the ORR onset allowing the mechanism to occur via the desirable 4 electron pathway, whilst also increasing the stability and methanol tolerance, compared to undoped graphene. This study clearly highlights the potential for heteroatom doped graphene to be a cost effect alternative to $\mathrm{Pt}$ based electrocatalysts, however by it utilising chemically exfoliated graphene oxide as the carbon precursor and implementing a chemical activation method, the resulting graphene based material is prone to restacking and crumpling of its sheets, which limits the stability and electrocatalytic potential of the material. Lin et $a .^{28}$ is not alone in their use of graphene oxide as a precursor, the vast majority of the literature utilises a similar fabrication methodology and thusly encounters the same detrimental effects.

In order to address the problem of restacking and crumpling graphene sheets, which is endemic within the literature, this paper presents a facile two-step methodology for the fabrication of nitrogen-doped nanoporous graphene. This involves, producing the nanoporous graphene via a chemical vapour deposition (CVD) method followed by introducing nitrogen groups via pyrolysis using ortho-phenylenediamine as a precursor. Table 1 summarises the current literature devoted to developing heteroatom-doped catalysts, which demonstrates that our approach is a novel methodology to realise nitrogen doped nanoporous graphene with outstanding electrochemical properties. The nitrogen doped nanoporous graphene electrocatalyst does not suffer from the restacking and crumpling problems associated with other heteroatom doped graphene based ORR electrocatalysts. This study therefore significantly increases the attractiveness of graphene-based electrocatalysts as potential cost-effective alternatives for Pt in PEMFCs.

\section{Experimental}

\section{Materials}

All chemical materials were used as received without further purification. Ortho-phenylenediamine (flaked 99.5\%), $\mathrm{H}_{2} \mathrm{SO}_{4}$ (98\%), $\mathrm{HNO}_{3}(70 \%), \mathrm{HCl}(37 \%)$, ethanol (99.8\%), and Nafion ( $5 \mathrm{wt} \%$ in lower aliphatic alcohols and water) were all purchased from Sigma Aldrich. Deionised water $(18 \mathrm{M} \Omega \mathrm{cm})$ was achieved by Millipore (Bedford, MA, USA) and used in all experiments.

\section{Preparation of nanoporous graphene}

Nanoporous graphene was synthesised by a chemical vapour deposition (CVD) process using methane as the carbon precursor. ${ }^{29}$ The reaction was carried out in a tubular quartz reactor $($ diameter $=5 \mathrm{~cm}$, length $=120 \mathrm{~cm}$ ) in the presence of hydrogen and nitrogen as the reducing and carrier gas, respectively. The optimum ratio of methane, hydrogen and nitrogen was found to be $4: 1: 1$. The CVD processes were conducted in a horizontal furnace at $900-1000{ }^{\circ} \mathrm{C}$ for $5-30$ minutes at a rate of $5{ }^{\circ} \mathrm{C} \mathrm{min}^{-1}$. Subsequently, the reactor was allowed to naturally cool to room temperature under a nitrogen atmosphere, after which the nanoporous graphene was collected. The obtained product was purified by refluxing in $20 \%$ HCL solution for 24 hours at room temperature. The purified sample was repeatedly washed and neutralised with deionized water and dried at $80{ }^{\circ} \mathrm{C}$ for 48 hours. The obtained product, typically $c a .2 \mathrm{~g}$ was oxidised via functionalization with a $200 \mathrm{~mL}$ mixture of concentrated sulfuric and nitric acids (3:1 $\mathrm{v} / \mathrm{v}$ ). The mixture was mechanically stirred and sonicated for 10 


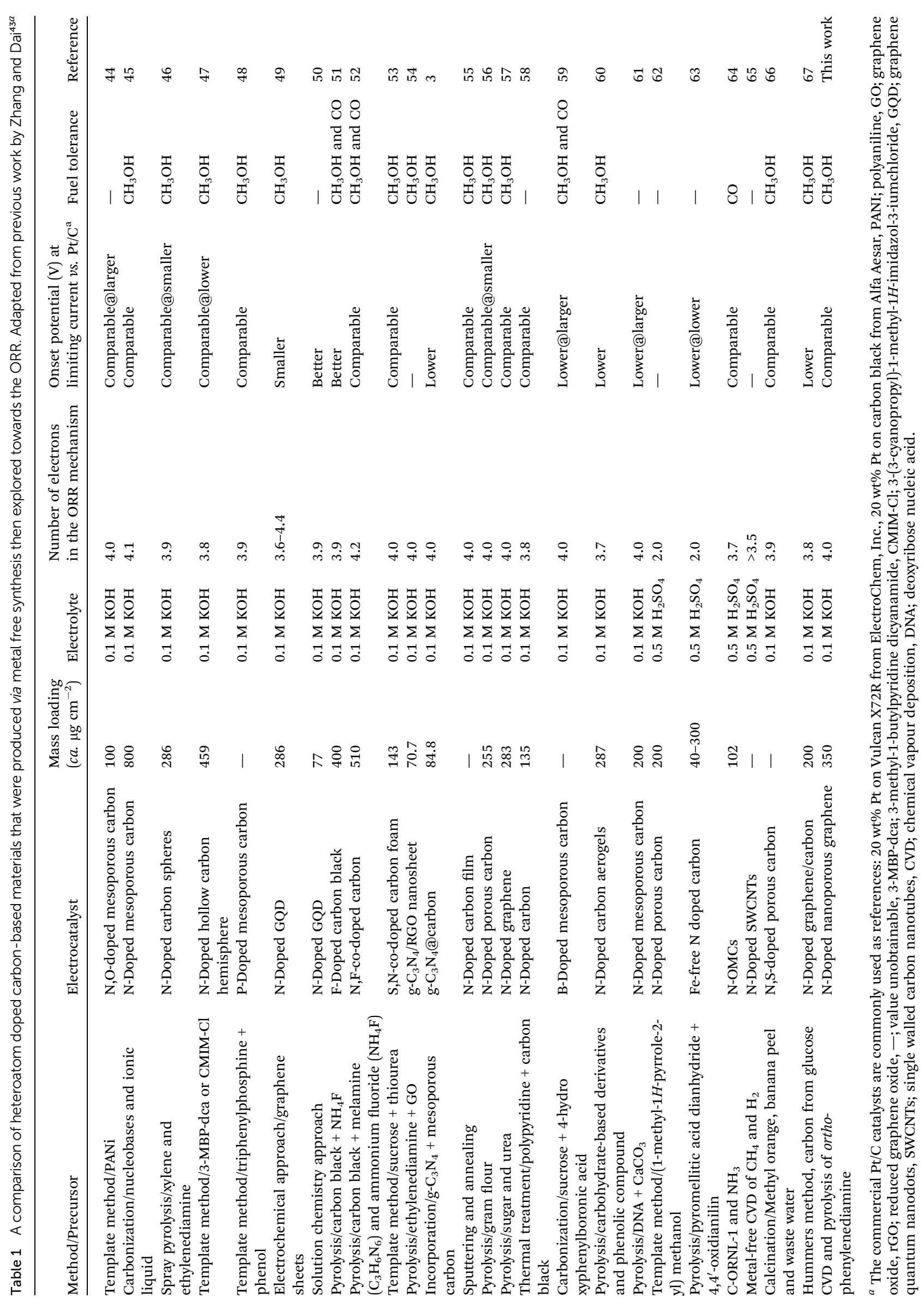


and 180 minutes, respectively. Next, the functionalised porous graphene was cooled down to room temperature and diluted with $500 \mathrm{~mL}$ deionised water. Subsequently, the solution was filtrated, neutralised and dried at $60{ }^{\circ} \mathrm{C}$ overnight. The oxidised porous graphene is denoted as PG herein.

\section{Nitrogen doped nanoporous graphene (NPG)}

Nitrogen doped nanoporous graphene was prepared using orthophenylenediamine (OPDA) as an aromatic nitrogen precursor. Initially, $0.2 \mathrm{~g}$ of the as-prepared NPG was ultrasonically dispersed in $200 \mathrm{~mL}$ ethanol. Different amounts of OPDA were then gradually added into separate suspensions of: $0.1,0.2,1$, and $2 \mathrm{~g}$ which are hereafter denoted as NPG 10.5, NPG 1-1, NPG 1-5, and NPG 1-10, respectively. The suspensions were further sonicated for 10 minutes in order to dissolve the OPDA. Next, the suspension was mechanically stirred (600 rpm) at room temperature for 6 hours and dried at $80{ }^{\circ} \mathrm{C}$ in order to allow complete solvent evaporation. The remnant solid was then collected and grinded with a pestle and mortar to obtain a uniform powder. Next, the obtained solid was placed into a quartz boat and pyrolyzed in a tubular furnace at $900{ }^{\circ} \mathrm{C}$ for 2 hours within a nitrogen atmosphere with a heating rate of $5{ }^{\circ} \mathrm{C} \mathrm{min}^{-1}$. The reactor was allowed cooled down to room temperature in the presence of nitrogen and the final product obtained was the NPG. As a control, oxidised nanoporous graphene was subjected to the same procedure without the addition of OPDA.

\section{Preparation of electrodes}

A rotating glassy carbon electrode (RGCE) (diameter: $3 \mathrm{~mm}$ ) was utilised in all electrochemical investigations. In each test, the RGCE was diligently polished using a microcloth and decreasing sizes of alumina. ( 0.3 and $0.05 \mu \mathrm{m}$ alumina slurry). The polished electrode was sequentially washed with deionised water and sonicated in ethanol to remove any residual alumina powder. For each electrocatalyst, $5 \mathrm{mg}$ powder was ultrasonically dispersed in $950 \mu \mathrm{L}$ of ethanol, ultrapure water $(1: 1 \mathrm{v} / \mathrm{v})$ and $50 \mu \mathrm{L}$ of Nafion solution (0.05 wt\%) for 15 minutes. Finally, $5 \mu \mathrm{L}$ of the as-prepared "ink" electrocatalysts were loaded upon the surface of the RGCE and allowed to dry at room temperature. The catalyst loading for all electrodes was estimated to correspond to $\mathrm{ca} .0 .35 \mathrm{mg} \mathrm{cm}^{-2}$.

\section{Physicochemical characterisation}

The crystallinity of the samples was investigated by X-ray diffraction (XRD) with a PW 1840 Philips device in the range of $10-90^{\circ}$ with the rate of $0.02(2 \theta / \mathrm{s})$. The morphology and elemental composition of the nanoporous graphene was examined by field emission scanning electron microscopy FEI Quanta 650F Environmental (SEM), energy-dispersive X-ray spectroscopy (EDX) and transmission electron microscopy (Tecnai G2 F20 S TWIN HR(S) (TEM), FEI). X-ray photoelectron spectroscopy (XPS) was performed using a hemispherical analyzer supplied by (XPS, SPECS Germany, PHOIBOS 150). The CasaXPS software (2.3.17PR1.1) was used for deconvolution of the XPS results by subtracting Shireley background. Raman spectra were carried out on an Almega Thermo Nicolet with an Ar ion laser source and excitation of $532 \mathrm{~nm}$. The Brunauer-Emmett-Teller (BET) and the BarrettJoyner-Halenda (BJH) methods were used for the estimation of the surface area and pores size distribution of the nanoporous graphene. The BET surface area, pore volume, and pore size distribution were assessed by nitrogen adsorption-desorption isotherms at $77 \mathrm{~K}$ utilizing an ASAP-2010 porosimeter (Micromeritics Corporation, GA).

\section{Electrochemical analysis}

Electrochemical analyses were performed using an Autolab potentiostat/galvanostat instrument (PGSTAT30 Eco Chemie, B.V, Netherlands), along with a rotating disk electrode (RDE) system in a conventional three electrode system. The RGCE was used as the working electrode, while $\mathrm{Ag} / \mathrm{AgCl}$ and $\mathrm{Pt}$ wire were used as the reference and counter electrode, respectively. Prior to electrochemical evaluation, including cyclic voltammetry (CV), linear sweep voltammetry (LSV), chronoamperometry (CA), and electrochemical impedance spectroscopy (EIS), of $0.1 \mathrm{M} \mathrm{KOH}$ were gently purged with pure oxygen or nitrogen for 30 minutes to get a saturated electrolyte. All potentials in this study are reported versus reversible hydrogen electrode (RHE).

\section{Results and discussion}

The overall fabrication process of the nitrogen doped nanoporous graphene (NPG) is summarised in Fig. 1 and detailed within the Experimental section. In the first step, the oxygen moieties on the nanoporous graphene likely attract the $-\mathrm{NH}_{2}$ in OPDA through electrostatic interactions, increasing the level of nitrogen doping. ${ }^{30}$ The decomposition of the adsorbed OPDA at $900{ }^{\circ} \mathrm{C}$ may also create new $\mathrm{C}-\mathrm{N}$ bonds in the nanoporous graphene lattice mainly as pyridinic, pyrrolic, and graphitic. The aromatic structure and the presence of two nitrogen atoms in the OPDA reactant leads to the production of nitrogen doped nanoporous graphene with a high content of nitrogen atoms.

\section{Physiochemical characterisation of the PG and NPG variants}

A full independent physicochemical characterisation was performed on the PG and NPG variants, this includes: Raman Spectroscopy, SEM, TEM, XPS and XRD. A brief summary of the characterisation is given below with interested readers directed to the ESI $\dagger$ where a full description of each physicochemical characterisation technique can be found.

Fig. $2 \mathrm{~A}$ and $\mathrm{S} 1 \uparrow$ show the SEM images and corresponding elemental mapping analysis of the PG and NPG 1-5. The 3D morphological structure can be observed to be one of high porosity for the both PG and NPG 1-5. The TEM images of the PG and NPG 1-5 were also taken to further investigate the crumpled, porous, and corrugated structure of PG and NPG 1-5 (see Fig. 2B and C). As presented in the TEM images, the more transparent and apparently fewer layers of the NPG 1-5, compared to PG, might be attributed to the nitrogen doping effect in agreement with the XRD and Raman results; this indicates that the NPG does not suffer from restacking and crumpling problems which is associated with other non-doped and other heteroatom doped graphenes. 

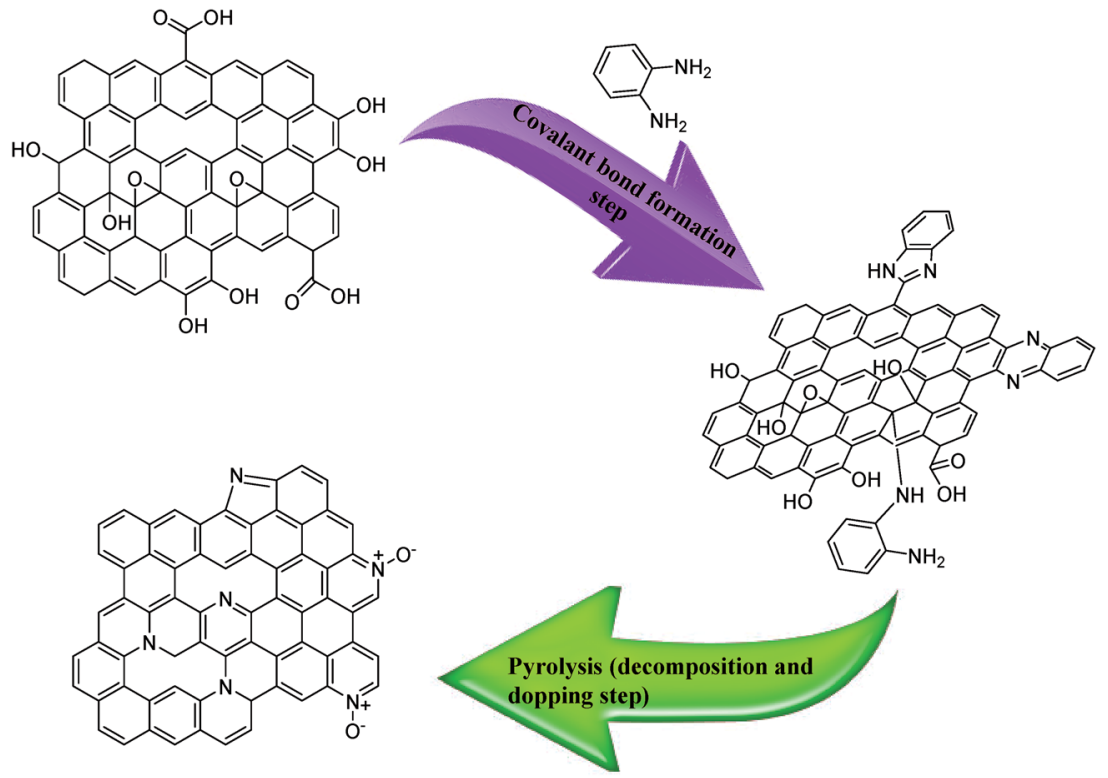

Fig. 1 Overview of the fabrication process to produce the nitrogen doped nanoporous graphene.

The interconnected framework and ultrathin nanosheets of the NPG 1-5 maintain lots of slit-shaped porous structures as the desirable sites for ORR. ${ }^{31}$ Simultaneously, the presence of amorphous carbon and highly graphitized structure of the NPG 1-5 can be obviously seen in the TEM images.
The XRD patterns of PG, NPG 1-0.5, NPG 1-1, NPG 1-10 and NPG 1-5 samples are shown within Fig. 2D. The XRD patterns reveal the (002) reflection of graphene structure at around $2 \theta \sim$ $29^{\circ}$ and $26^{\circ}$ for non-doped and nitrogen doped samples, respectively, suggesting that nanoporous graphene exists in single or few
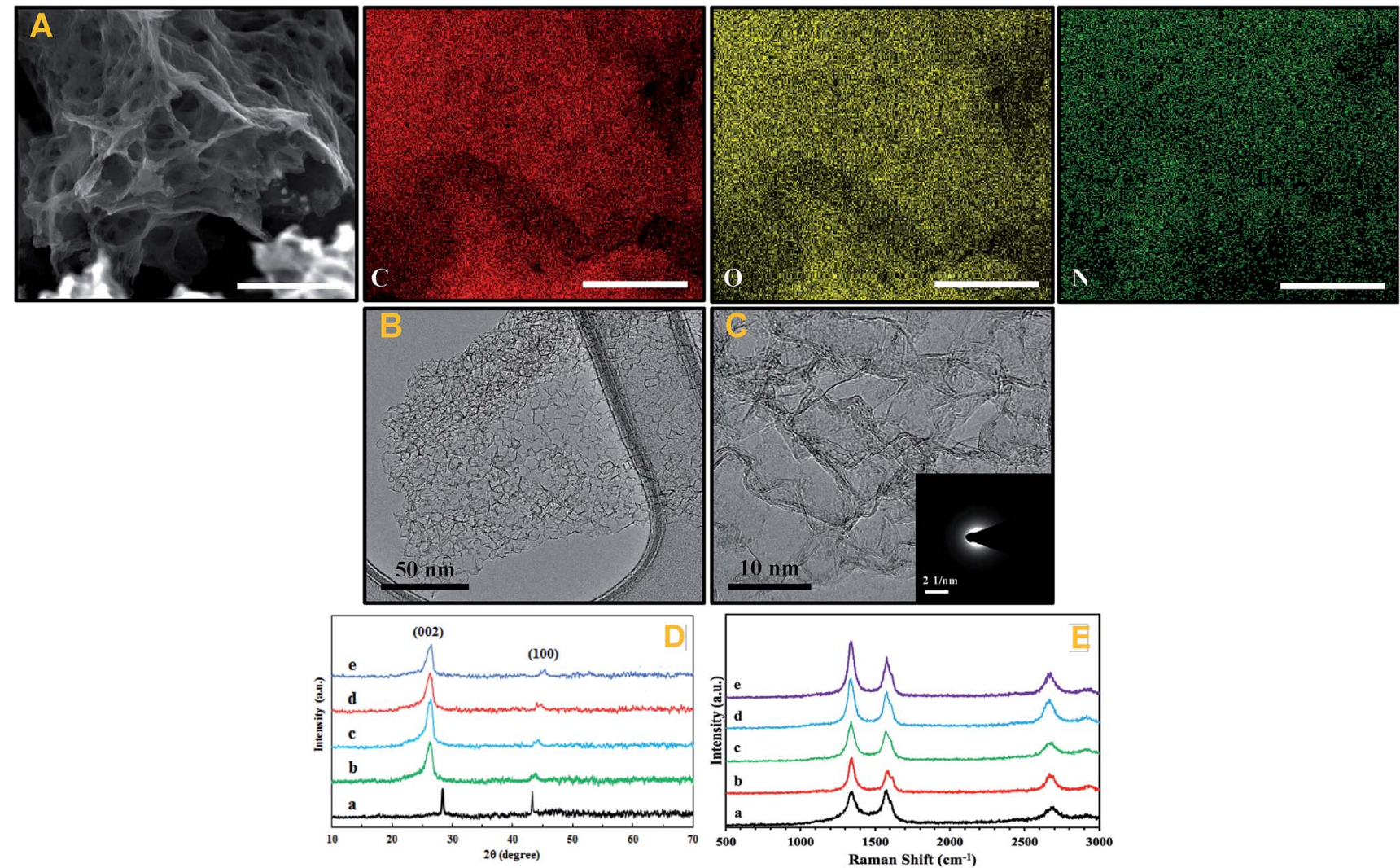

Fig. 2 (A) SEM images and EDX elemental mapping analysis of NPG 1-5 (scale bar is $2 \mu \mathrm{m}$ ), (B) low resolution TEM image of NPG 1-5, (C) high resolution TEM image of NPG 1-5 (SAED inset), (D) XRD patterns and (E) Raman spectra of (a) PG, (b) NPG 1-0.5, (c) NPG 1-1, (d) NPG 1-10, (e) NPG 1-5. 
layers. The scattered peaks at $c a .44-45^{\circ}$ can be assigned to the (100) crystal plane of graphite, implying the presence of both crystalline and amorphous carbon structures. ${ }^{32}$

As observed, the strong peaks of nitrogen doped nanoporous graphene are broadened and shifted toward lower angles in comparison with non-doped nanoporous graphene, indicating that the pyrolysis treatment with OPDA moderately disorders the graphene framework, possibly due to the creation of more defects resulting from heteroatom doping effect. ${ }^{33}$ The number of graphene sheets can be estimated by calculating the interlayer spacing of the lattices and the mean crystallite size of the powder, which is obtained by the Scherrer equation: ${ }^{34}$ $L_{h k l}=\frac{0.9 \lambda}{\beta \cos \theta}$ where $L_{h k l}$ is the mean crystallite size of powder for the related Miller indices $(h k l), \lambda$ is the wavelength of radiation (1.542 $\AA$ ), $\beta$ is the full widths at half maximum (FWHM) in radians, and $\theta$ is the scattering angle. The interlayer spacing of graphene sheets can be calculated by Bragg's law: ${ }^{34}$ $d_{h k l}=\frac{n \lambda}{2 \sin \theta}$ where $d_{h k l}$ is the interlayer spacing of the lattices for the related Miller indices $(h k l)$ and $n$ represents an integer for the order of the diffraction peak. Accordingly, the number of graphene layers can be estimated trough the following equation: ${ }^{34} N=\left(\frac{L}{d}\right)_{h k l}$. The number of layers, FWHM, $L_{h k l}$, and $d_{h k l}$ are summarized in Table 2 (main paper) with respect to the XRD spectra.

The number of graphene sheets for PG and NPG 1-0.5 was estimated by calculating the interlayer spacing of the lattices and the mean crystallite size of the powder and found to be 7 and 5, respectively. Whilst the number of graphene layers for NPG 1-1, NPG 1-5, and NPG 1-10 is equal to 4 (see Table 2). The decreasing number of graphene layers can be attributed to increasing the space layer for nitrogen doped nanoporous samples versus un-doped nanoporous graphene.

The augmentation of FWHM quantities of nitrogen doped graphene compared to the pristine nanoporous graphene further verifies the successful doping of $\mathrm{N}$ atoms into the carbon skeleton.

Furthermore, by increasing the OPDA concentration, the (002) peaks get somewhat broader from NPG 1-0.5 to NPG 1-5 (FWHM in Table 2), signifying that the higher concentration of OPDA leads to the creation of more disorders in the graphene network. However, further increasing the concentration of OPDA to NPG 1-10 reduces the FWHM, suggesting the NPG 1-5 as the optimized ratio for the preparation of nitrogen doped nanoporous graphene.

Table 2 XRD specification of PG and NPG

\begin{tabular}{llllll}
\hline Sample & $2 \theta\left(^{\circ}\right)$ & FWHM $\left(^{\circ}\right)$ & $L_{h k l}(\mathrm{~nm})$ & $d_{h k l}(\AA)$ & $\begin{array}{l}\text { Number of } \\
\text { layers } \approx\end{array}$ \\
\hline PG & 29.01 & 4.22 & 1.95 & 3.26 & $6-7$ \\
NPG 1-0.5 & 26.65 & 5.78 & 1.41 & 3.34 & $4-5$ \\
NPG 1-1 & 26.58 & 5.91 & 1.38 & 3.36 & $3-4$ \\
NPG 1-10 & 26.54 & 5.95 & 1.37 & 3.36 & $3-4$ \\
NPG 1-5 & 26.50 & 6.12 & 1.33 & 3.37 & $3-4$
\end{tabular}

The Raman spectra of the samples are presented in Fig. 2E. The three expected characteristic peaks are observed at $c a .1350$, 1580 , and $2685 \mathrm{~cm}^{-1}$ for all samples correspond to D, G, and $2 \mathrm{D}$ bands, respectively (see Table 3 ). The three distinctive peaks at around 1350, 1580, and $2685 \mathrm{~cm}^{-1}$ for all samples correspond to $\mathrm{D}, \mathrm{G}$, and 2D bands, respectively (Table 3 ). The $\mathrm{G}$ band is considered as the first-order scattering of the $\mathrm{E}_{2 \mathrm{~g}}$ mode of $\mathrm{sp}^{2}$ carbon atoms in graphene matrix, whereas the $\mathrm{D}$ band is related to the breathing mode of $\mathrm{A}_{1 \mathrm{~g}}$ symmetry, indicating the presence of defects, microstructure disordering and amorphous carbon. ${ }^{35}$ Commonly, the $I_{\mathrm{D}} / I_{\mathrm{G}}$ reveals the information about the relation of the $\mathrm{sp}^{3}$ hybridized carbon (disordered structures) to $\mathrm{sp}^{2}$ hybridized carbon (graphitic structures). It should be taken into account that the higher ratio of $I_{\mathrm{D}} / I_{\mathrm{G}}$ signifies the higher in plane and edge defects emanating from doping, functionalization and exfoliation of graphene. ${ }^{36}$ Obviously, the intensity of D and G band of PG is approximately equal, suggesting the presence of crystallite and amorphous structures in nanoporous graphene at the same time. The $I_{\mathrm{D}} / I_{\mathrm{G}}$ ratio of PG is about 0.93 , while doping nitrogen increases the amount of $I_{\mathrm{D}} / I_{\mathrm{G}}$ ratio to $1.1,1.23,1.42$, and 1.54 for NPG 1-0.5, NPG 1-1, NPG 110 , and NPG 1-5, respectively (Table 3 ). The augmentation of $I_{\mathrm{D}} /$ $I_{\mathrm{G}}$ can be attributed to the disruption of aromatic $\pi-\pi$ electrons in the graphene matrix confirming successful nitrogen doping procedure. The $I_{\mathrm{D}} / I_{\mathrm{G}}$ ratio increases by increasing the concentration of OPDA in the ratio of $(1: 5)$, despite higher concentrations of OPDA $(1: 10)$ reducing the of $I_{\mathrm{D}} / I_{\mathrm{G}}$ ratio. This indicates NPG 1-5 is the best proportion. Apart from that, the shape and intensity of the $2 \mathrm{D}$ bands are known as one of the most prominent features of Raman spectra for identifying the number of layers in two-dimension carbon nanostructures. As the $2 \mathrm{D}$ band gets broader and positively shifted toward higher wave number, the number of graphene layers increases. ${ }^{37}$ The $2 \mathrm{D}$ bands of nitrogen doped porous graphene are partially sharper than that of non-doped porous graphene (Fig. 2E), indicating that the number of graphene layers can be decreased via nitrogen doping. However, as the XRD results showed, there is no significant alteration between the number of graphene layers before and after nitrogen doping. Also, the intangible augmentation of the $I_{2 \mathrm{D}}$ by introducing nitrogen atoms (Table 3 ) leads to the increase in the $I_{2 \mathrm{D}} / I_{\mathrm{G}}$ ratio, which further verifies the diminishing the number of graphene sheets. It can be concluded that the nitrogen doped samples are composed of few-layered graphene as well as sufficient defects as the desirable sites for oxygen reduction reaction.

XPS was performed to investigate the elemental composition of the electrocatalysts and verify the degree and type of nitrogen doping. The XPS surveys of porous graphene presented in Fig. S2 and S3† shows two distinctive peaks at 285 and $531 \mathrm{eV}$ corresponding to $\mathrm{C} 1 \mathrm{~s}$ and $\mathrm{O} 1 \mathrm{~s}$, respectively, without any other impurities. In the NPG spectra, the appearance of the characteristic peaks at about $400 \mathrm{eV}$ confirms the successful doping of nitrogen atoms in graphene matrix. Last, BET surface area $\left(\mathrm{m}^{2}\right.$ $\left.\mathrm{g}^{-1}\right)$, pore volume $\left(\mathrm{cm}^{3} \mathrm{~g}^{-1}\right)$ and mean pore size $(\mathrm{nm})$ for PG sample was determined to correspond to 721.3, 2.3 and 12.6, respectively (see ESI and Fig. S4 $\dagger$ ). Also, the BET surface area $\left(\mathrm{m}^{2} \mathrm{~g}^{-1}\right)$, pore volume $\left(\mathrm{cm}^{3} \mathrm{~g}^{-1}\right)$ and mean pore size $(\mathrm{nm})$ for 
Table 3 Raman specification of the PG and NPG

\begin{tabular}{|c|c|c|c|c|c|c|c|c|}
\hline Sample & $\mathrm{D}$ band $\left(\mathrm{cm}^{-1}\right)$ & G band $\left(\mathrm{cm}^{-1}\right)$ & 2D band $\left(\mathrm{cm}^{-1}\right)$ & $I_{\mathrm{D}}$ & $I_{\mathrm{G}}$ & $I_{\mathrm{D}} / I_{\mathrm{G}}$ & $I_{2 \mathrm{D}}$ & $I_{2 \mathrm{D}} / I_{\mathrm{G}}$ \\
\hline PG & 1351.8 & 1578.3 & 2673.6 & 327.9 & 350.7 & 0.93 & 189.7 & 0.55 \\
\hline NPG 1-0.5 & 1350.6 & 1580.97 & 2681.1 & 348.1 & 316.8 & 1.10 & 195.1 & 0.62 \\
\hline NPG 1-10 & 1351.6 & 1582.5 & 2683.2 & 497.5 & 352.6 & 1.42 & 222.4 & 0.63 \\
\hline NPG 1-5 & 1353.4 & 1582.3 & 2685.2 & 544.6 & 354.1 & 1.54 & 225.1 & 0.64 \\
\hline
\end{tabular}

NPG 1-5 sample is $665.2,1.8$ and 13.7 , respectively. In addition to BET surface area, the pore volume and pore size of the PG and NPG 1-5 remains approximately unchanged, indicating that nitrogen doping does not principally deteriorate the mesoporous structure of porous graphene, which is in good agreement with TEM images and Raman spectra. Thus, the PG and NPG variants utilised throughout this study have been fully characterised and revealed to be of high quality and can be accurately described as such.

\section{Electrochemical evaluation of ORR performance}

Initially it was vital to assess the intrinsic electrochemical response of the NPG variants in $\mathrm{N}_{2}$ saturated $0.1 \mathrm{M} \mathrm{KOH}(50 \mathrm{mV}$ $\mathrm{s}^{-1}$ ) to ensure that there was no visible redox peaks within the potential region where the $\mathrm{O}_{2}$ reduction peak is expected, as this could result in convolution of the observed signal output. Fig. S5 $\uparrow$ shows that all of the NPG variants voltammetric profiles that indicate for this electrode size/configuration there are no noticeable peaks. Upon $\mathrm{O}_{2}$ saturation of the $0.1 \mathrm{M} \mathrm{KOH}$ welldefined redox peaks are clearly visible for the NPG variant indicating an efficient catalyst towards the ORR. It can be observed in Fig. $55 \dagger$ that the obtained potential peak and cyclic voltammograms area increases with increasing concentrations of OPDA, i.e. more nitrogen doping; the improvements in the voltammetry can be attributed to the formation of more graphitic and pyridinic ORR active sites. It is clear upon inspection of this figure and Table 5 that the NPG variants all have a more electropositive ORR onset potential at $c a .+1.0 \mathrm{~V}(v s$. RHE), than the un-doped PG, which has an ORR onset of $+0.82 \mathrm{~V}$ (vs. RHE). It is therefore clear that nitrogen doping the PG results in a significant increase in its ORR electrocatalytic activity. Of particular interest is the comparability of the NPG variants (especially NPG $1-5$, which exhibited the most electropositive ORR onset potential at $1.03 \mathrm{~V}$ ( $v s$. RHE)) ORR onset potentials to the industrial standard of $\mathrm{Pt} / \mathrm{C} 20 \%$, which was observed to have an ORR onset of $1.05 \mathrm{~V}$ (vs. RHE). This clearly indicates the potential for an optimised NPG material to act as a cost effective alternative to $\mathrm{Pt} / \mathrm{C}$ as a cathodic electrode material within PEMFCs.

The CV profiles (in $\mathrm{O}_{2}$ saturated $0.1 \mathrm{M} \mathrm{KOH}$ ) of the optimized nitrogen doped porous graphene (NPG 1-5), bare/unmodified PG and commercialised Pt/C 20\% are shown in Fig. 3 for comparative purposes. As stated above the ORR onset potential for the NPG $1-5$ is more electropositive than the bare/ unmodified PG and comparable to that of Pt/C 20\%. Upon inspection of this figure it is also evident that NPG 1-5 has a larger peak potential than either the bare/unmodified PG or $\mathrm{Pt} / \mathrm{C} 20 \%$. This supports the interference that N doping the PG increases its catalytic activity towards the ORR. In order to assess the limiting current and whether there is a change in the ORR mechanism associated with the observed increase in the ORR, we employed rotating disk electrochemistry as is common within the literature. This was performed by carrying out LSV in $\mathrm{O}_{2}$ saturated $0.1 \mathrm{M} \mathrm{KOH}$ at scan rate of $5 \mathrm{mV} \mathrm{s}{ }^{-1}$, utilising various rotation rates. The LSV plots at the rotation rates from 250 to $3500 \mathrm{rpm}$ show that by increasing the rotation rate, the limiting current increases as expected (see Fig. S6, $\dagger$ with a comparison of the LSVs at $1500 \mathrm{rpm}$ being given in Fig. 4b). As presented, for all of the nitrogen doped samples, the ORR
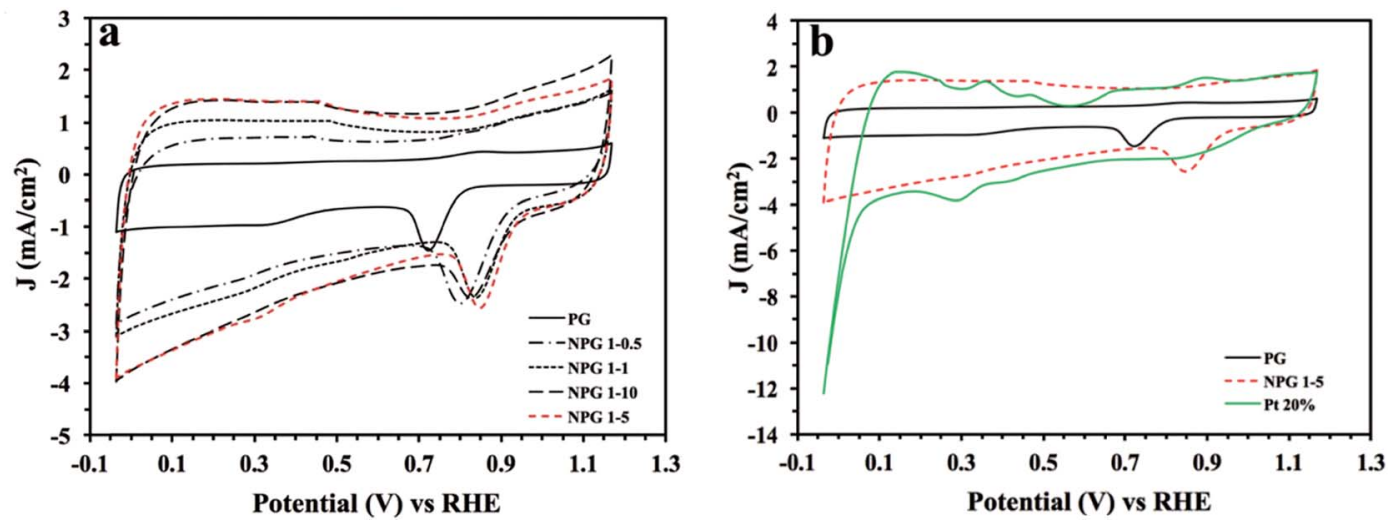

Fig. 3 (a) CVs of PG and the variants and (b) optimised NPG 1-5 compared against Pt/C 20\% and PG. Solution composition: $0.1 \mathrm{M} \mathrm{KOH}$ (O 2 saturated), scan rate $50 \mathrm{mV} \mathrm{s}^{-1}$ (vs. RHE). 

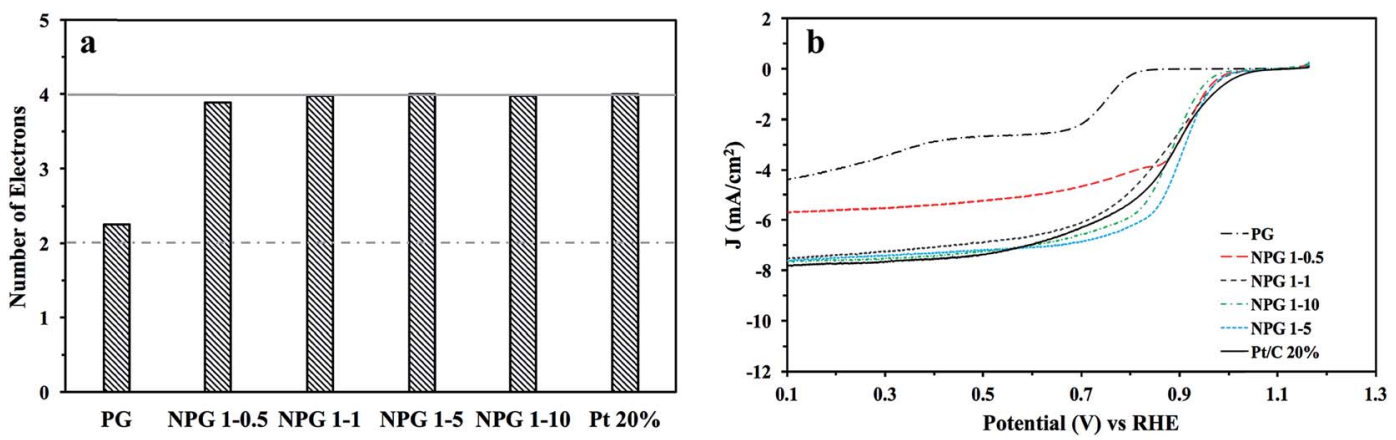

Fig. 4 (a) Plot for the number of electron transferred for each sample. Also shown for convenience is a dashed and solid line showing the 2electron and 4-electron transfer processes; the 4-electrode process is the more desirable indicating an efficient electrocatalyst. (b) Comparison of linear sweep voltammetry recorded in alkaline media at $1500 \mathrm{rpm}$.

current density initiates at $c a .0 .82-1.05 \mathrm{~V}$ and then reaches a limiting current density at $c a .0 .7-0.8 \mathrm{~V}$, indicating high ORR activity. Among all nitrogen doped nanoporous electrocatalysts, NPG 1-5 possesses the highest limiting current of $c a .7 .8 \mathrm{~mA}$ $\mathrm{cm}^{-2}$. In order to deduce the ORR mechanism the number of electron transfers, $n$, participating in the electrochemical reaction need to be deduced. A commonly employed approach is to calculate this via rotating disc analysis via the Koutecky-Levich equation: ${ }^{10-12,38} \quad \frac{1}{J}=\frac{1}{J_{\mathrm{K}}}+\frac{1}{J_{\mathrm{d}}}=\frac{1}{J_{\mathrm{K}}}+\frac{1}{B \omega^{0.5}} \quad$ where $\quad B=$ $0.62 n F D^{2 / 3} v^{-1} / 6 C_{0}$. In these equations, $J$ is the experimental current density, $J_{\mathrm{K}}$ is the kinetic-limiting current density, $J_{\mathrm{d}}$ is the diffusion-limiting current density, $\omega$ is the rotation speed (rpm), $n$ is the electron transfer number in ORR, $F$ is the Faraday constant $\left(96485 \mathrm{C} \mathrm{mol}^{-1}\right), D$ is the diffusion coefficient of $\mathrm{O}_{2}$ in $0.1 \mathrm{M}$ KOH solution $\left(1.9 \times 10^{-5} \mathrm{~cm}^{2} \mathrm{~s}^{-1}\right), v$ is the electrolyte velocity $\left(0.01 \mathrm{~cm}^{2} \mathrm{~s}^{-1}\right)$ and $C_{0}$ is the bulk concentration of $\mathrm{O}_{2}$ $\left(1.2 \times 10^{-6} \mathrm{~mol} \mathrm{~cm}^{-3}\right)$. The Koutecky-Levich (K-L) plots of $J^{-1}$ versus $\omega^{-1}$ at various potentials (RHE) from RDE measurements exhibit straight and parallel lines, implying the first-order reaction (See Fig. S7†). Analysis of the K-L slope plots for the PG suggest that ORR mechanism occurs via a two electron pathway, whilst the nitrogen doped samples where observed to have an ORR mechanism that occurred by the desirable fourelectron pathway, suggesting the almost complete $\mathrm{O}_{2}$ reduction to $\mathrm{OH}^{-}$. The exact number electrons involved in the $\mathrm{PG}$ and NPG variants reaction mechanism are displayed within Fig. 4a and Table 5. It can be inferred that the doping of PG with $\mathrm{N}$ increases the ORR activity by enabling the reaction mechanism to occur by the desirable 4 electron pathway.
The obtained results suggest that the high electrocatalytic activity of the NPG can be attributed to the degree and type of nitrogen doping. Whilst the detailed role of different nitrogen containing phases is still unclear, ${ }^{39}$ with various studies within the literature offering alternative theories. For example Lai et $a l^{40}$ reported that the graphitic nitrogen phase determines the limiting current density, while the pyridinic phase has an undeniable role in onset potential improvement. ${ }^{40}$ Contrary to this, it has been reported that the high electrocatalytic activity of nitrogen doped carbon nanostructures can be attributed to pyrrolic nitrogen content. ${ }^{39}$ It was therefore important to assess the surface groups present on the NPG samples to determine their involvement in observed ORR activity. It was initially theorised as the incorporation of OPDA increased the percentage of nitrogen groups present on the graphene's surface would increase, however, according to the XPS results, the coverage of graphitic nitrogen remained almost unchanged with increasing usage of OPDA. This suggests that the electocatalytic activity can be much more affected by pyridinic nitrogen as the favourable active sites for ORR rather than the graphitic nitrogen. The pyridinic percentage in NPG $1-5$ is higher than other N-doped electrocatalysts, possibly explaining why it displayed the most beneficial ORR activity. The pyridinic nitrogen doping into graphene network can increase the $2 \mathrm{p} \pi$ state of $\mathrm{C}-\mathrm{N}$, as well as, weaken the $\mathrm{C}-\mathrm{C}$ electron density. ${ }^{41}$ Moreover, the increase in N/C ratio (Table 4) would alter the electronic density of state close to the Fermi level, which enhances the adsorption of $\mathrm{O}_{2}$ and facilitates the electron transfer between catalyst and oxygen molecules. ${ }^{17,41}$ Thus, the best electrocatalytic performance of NPG 1-5 among the other

Table 4 Atomic composition of the various samples obtained from XPS analysis

\begin{tabular}{|c|c|c|c|c|c|c|c|c|}
\hline \multirow[b]{2}{*}{ Sample } & \multirow{2}{*}{$\frac{\text { Atomic percentages }(\%)}{\mathrm{C}}$} & \multirow[b]{2}{*}{$\mathrm{O}$} & \multirow[b]{2}{*}{$\mathrm{N}$} & \multirow[b]{2}{*}{$\mathrm{N} / \mathrm{C}$} & \multicolumn{4}{|c|}{ Nitrogen composition atomic percentages (\%) } \\
\hline & & & & & Pyridinic N & Pyrrolic N & Graphitic N & Oxidized $\mathrm{N}$ \\
\hline NPG 1-0.5 & $92.1 \pm 3.2$ & $3.8 \pm 0.1$ & $4.1 \pm 0.1$ & 0.045 & $15.2 \pm 0.6$ & $24.7 \pm 1.3$ & $40.3 \pm 2.2$ & $19.8 \pm 0.7$ \\
\hline NPG 1-1 & $91.5 \pm 3.9$ & $2.2 \pm 0.1$ & $6.3 \pm 0.2$ & 0.069 & $25.4 \pm 1.3$ & $12.1 \pm 0.4$ & $45.2 \pm 1.8$ & $17.3 \pm 1.1$ \\
\hline NPG 1-10 & $90.4 \pm 2.7$ & $1.9 \pm 0.1$ & $7.7 \pm 1.2$ & 0.085 & $27.4 \pm 3.6$ & $10.0 \pm 1.7$ & $44.4 \pm 2.7$ & $18.2 \pm 0.8$ \\
\hline
\end{tabular}


Table 5 Comparison between the number of electron transferred in the ORR, onset potential and current density

\begin{tabular}{llcl}
\hline Samples & $\begin{array}{l}\text { Number of electrons } \\
\text { transferred }(n)\end{array}$ & $\begin{array}{l}\text { Steady state current } \\
\text { density }\left(\mathrm{mA} \mathrm{cm} \mathrm{cm}^{-2}\right)\end{array}$ \\
\hline PG & 2.2 & +0.82 & 4.52 \\
NPG 1-0.5 & 3.8 & +1.00 & 5.79 \\
NPG 1-1 & 3.9 & +1.01 & 7.31 \\
NPG 1-5 & 4.0 & +1.03 & 7.42 \\
NPG 1-10 & 4.0 & +0.99 & 7.45 \\
Pt/C 20\% & 4.0 & +1.05 & 7.61
\end{tabular}

electrocatalysts can be attributed to it having the highest percentage of pyridinic content (33.5\%).

\section{Stability and methanol tolerance of the NPG variants}

The tolerance of the electrocatalysts towards methanol crossover was evaluated by CV and chronoamperometry (CA). Fig. S5 $\dagger$ shows the typical CV response obtained for the NPG variants and $\mathrm{Pt} / \mathrm{C} 20 \%$ when $3 \mathrm{M}$ methanol was introduced to an oxygenated solution of $0.1 \mathrm{M} \mathrm{KOH}$. It is clear that the addition of methanol does not significantly alter the $\mathrm{CV}$ profiles on the nitrogen doped samples and PG, implying promising selectivity toward ORR against methanol oxidation, whereas methanol oxidation leads to the appearance of large anodic peak at $\mathrm{Pt} / \mathrm{C}$ electrode showing the poisoning effect upon fuel crossover. The stability of the electrocatalysts upon addition of methanol was further confirmed by CA measurements through applying a constant potential $(1.0 \mathrm{~V} v$ s. RHE) and a rotation of $1500 \mathrm{rpm}$, in $\mathrm{O}_{2}$ saturated electrolyte, for a duration of 1000 seconds (see Fig. S8†). An appropriate stability, as well as an excellent methanol tolerance of the nitrogen doped samples, can be observed. The loss of current density of the nitrogen doped samples was estimated to be less than $15 \%$ after addition of $3 \mathrm{M}$ methanol at $500^{\text {th }}$ second. In the case of PG, the loss of current density is more than nitrogen doped samples, accentuating the role of nitrogen atoms and porous structure on the stability of the electrocatalysts for ORR. According to Fig. S8f, $\uparrow$ the Pt/C electrocatalyst shows a significant current decline of about $73 \%$ compared to nitrogen doped, representing a rapid poisoning of the platinum active sites, with minimal restoration of the current density over the remaining duration. The longterm durability of the electrocatalysts were examined by exposing the electrode to equal conditions for $c a .27$ hours. As observed in Fig. S9(a), $\uparrow$ the nitrogen doped samples display remarkable stability over the duration of the experiment, this is due to the slower attenuation of current density compared to PG and $\mathrm{Pt} / \mathrm{C}$. The CA responses and the corresponding normalised current is shown in Fig. S9(b). $\dagger$ The normalized current also indicates the higher stability of the nitrogen doped electrocatalysts compared to Pt/C. The results show that the asprepared metal free catalysts not only possess high selectivity toward oxygen reduction, but also have outstanding stability in comparison to $\mathrm{Pt} / \mathrm{C}$.

Electrochemical impedance spectroscopy (EIS) was employed to further elucidate the electrocatalytic performance of the samples. Nyquist plots of the samples and $\mathrm{Pt} / \mathrm{C}$ at different potential are presented in Fig. S10. $\dagger$ The Nyquist curves show an arc-like or semicircle profile with different diameter corresponding to the charge transfer resistance $\left(R_{\mathrm{ct}}\right)$. For all of the nitrogen doped samples and $\mathrm{Pt} / \mathrm{C}$, the diameter of the semicircles shrinks by decreasing the applied potential to $+0.87 \mathrm{~V}$ (vs. RHE) and slightly increases at $+0.82 \mathrm{~V}$, indicating higher electrical conductivity in the vicinity of $+0.865 \mathrm{~V}$ ( $v s$. RHE), which is close to the peak potential in cyclic voltammetry. A similar trend does is not observed for the non-doped porous graphene, further highlighting the role of nitrogen atoms and high specific surface area, which facilitates electron transfer in the conductive channels of NPG. The comparison of Nyquist plots at $+0.87 \mathrm{~V}$ ( $v s$. RHE) and the corresponding equivalent circuit are shown in Fig. S11. $\dagger$ Based on the equivalent circuit fitting, the $R_{\mathrm{ct}}$ of PG, NPG 1-0.5, NPG 1-1, NPG 1-10, NPG 1-5, and $\mathrm{Pt} / \mathrm{C}$ were calculated to be $2335,283,255,212,155$, and 496 $\Omega$, respectively. The semicircle diameter of NPG 1-5 is slightly smaller than other samples. The $R_{\text {ct }}$ of all nitrogen doped samples are noticeably lower than that of PG and Pt/C, suggesting improved electron and ion transfer between electrode and electrolyte. Prior literature has demonstrated that the nitrogen atoms, especially pyridinic groups, next to the carbon atoms provide beneficial electrochemical/electrocatalytic sites due to their localised density of states in the occupied region near the Fermi level. ${ }^{41}$ Moreover, density functional theory (DFT) indicates that carbon atoms in the vicinity of the pyridinic nitrogen can act as Lewis bases because of its tendency to donate the electron pair. ${ }^{42}$ Therefore, in accordance to the XPS results, the high concentration of pyridinic nitrogen's of the electrocatalysts (i.e. NPG 1-5 and NPG 1-10, Table 5) result in enhanced electron transport and reduced charge transfer resistance.

In summary, this study has shown that by producing nitrogen doped nanoporous graphene, via a CVD method followed by introducing nitrogen groups via pyrolysis using orthophenylenediamine as a precursor, a stable and cost effective alternative to the $\mathrm{Pt} / \mathrm{C}$ can be fabricated for use as a cathodic material in a PEMFC.

\section{Conclusions}

NPG electrocatalysts have been successfully prepared and are shown to be useful electrocatalysts towards the ORR with comparable electrocatalytic activity to a commercially available Pt/C. Table 1 provides a thorough literature overview of 
nitrogen-doped electrocatalysts, where it can be seen that our fabrication approach is novel with the electrochemical performance demonstrated to be at least comparable, if not better than those reported within Table 1 . The outstanding electrocatalytic activity of the nitrogen doped electrocatalysts are due to its high surface area, high nitrogen content, large amount of defects and a nanoporous structure; the NPG does not suffer from restacking and crumpling problems. The homogenously distributed $\mathrm{N}$-species not only provide numerous ORR active sites, including graphitic and pyridinic phases, but also contribute to the highly disordered and nanoporous structure, all of which enhances electron transfer processes between the NPG and oxygen. Furthermore, the long-term stability and methanol crossover effect of the optimised electrocatalyst $(16 \%$ signal output loss) towards the ORR is optimal over that of $\mathrm{Pt} / \mathrm{C}$ (73\% signal output loss). In summary, the prepared electrocatalysts have potential as an excellent alternative to precious metal based catalysts for oxygen reduction reaction.

\section{Conflicts of interest}

There are no conflicts to declare.

\section{Acknowledgements}

The authors would like to thank Catalan Institute of Nanoscience and Nanotechnology (ICN2) for XPS measurements and HRTEM images. Funding from the Engineering and Physical Sciences Research Council (Reference: EP/N001877/1), British Council Institutional Grant Link (No. 172726574) is acknowledged. The Manchester Fuel Cell Innovation Centre is funded by the European Regional Development Fund.

\section{References}

1 G. Wu, K. L. More, C. M. Johnston and P. Zelenay, HighPerformance Electrocatalysts for Oxygen Reduction Derived from Polyaniline, Iron, and Cobalt, Science, 2011, 332(6028), 443-447.

2 B. C. H. Steele and A. Heinzel, Materials for fuel-cell technologies, Nature, 2001, 414(6861), 345-352.

3 Y. Zheng, Y. Jiao, J. Chen, J. Liu, J. Liang, A. Du, W. Zhang, Z. Zhu, S. C. Smith, M. Jaroniec, G. Q. Lu and S. Z. Qiao, Nanoporous Graphitic-C3N4@Carbon Metal-Free Electrocatalysts for Highly Efficient Oxygen Reduction, $J$. Am. Chem. Soc., 2011, 133(50), 20116-20119.

4 J. H. Zagal and M. T. M. Koper, Reactivity Descriptors for the Activity of Molecular MN4 Catalysts for the Oxygen Reduction Reaction, Angew. Chem., Int. Ed., 2016, 55(47), 14510-14521.

5 S. J. Rowley-Neale, D. A. C. Brownson, J. M. Fearn, G. C. Smith, X. Ji and C. E. Banks, 2D Molybdenum Disulphide (2D-MoS2) Modified Electrodes Explored Towards the Oxygen Reduction Reaction, Nanoscale, 2016, 8, 14767-14777.

6 L. Bu, S. Guo, X. Zhang, X. Shen, D. Su, G. Lu, X. Zhu, J. Yao, J. Guo and X. Huang, Surface engineering of hierarchical platinum-cobalt nanowires for efficient electrocatalysis, Nat. Commun., 2016, 7, 11850.

7 N. S. Porter, H. Wu, Z. Quan and J. Fang, Shape-Control and Electrocatalytic Activity-Enhancement of Pt-Based Bimetallic Nanocrystals, Acc. Chem. Res., 2013, 46(8), 1867-1877.

8 L. Lin, Q. Zhu and A.-W. Xu, Noble-Metal-Free Fe-N/C Catalyst for Highly Efficient Oxygen Reduction Reaction under Both Alkaline and Acidic Conditions, J. Am. Chem. Soc., 2014, 136(31), 11027-11033.

9 K. Gong, F. Du, Z. Xia, M. Durstock and L. Dai, Nitrogendoped carbon nanotube arrays with high electrocatalytic activity for oxygen reduction, Science, 2009, 323(5915), 760764.

10 L. Feng, Y. Yan, Y. Chen and L. Wang, Nitrogen-doped carbon nanotubes as efficient and durable metal-free cathodic catalysts for oxygen reduction in microbial fuel cells, Energy Environ. Sci., 2011, 4(5), 1892-1899.

11 L. Qu, Y. Liu, J.-B. Baek and L. Dai, Nitrogen-doped graphene as efficient metal-free electrocatalyst for oxygen reduction in fuel cells, ACS Nano, 2010, 4(3), 1321-1326.

12 D. Geng, Y. Chen, Y. Chen, Y. Li, R. Li, X. Sun, S. Ye and S. Knights, High oxygen-reduction activity and durability of nitrogen-doped graphene, Energy Environ. Sci., 2011, 4(3), 760-764.

13 Z. Lin, G. Waller, Y. Liu, M. Liu and C.-P. Wong, Facile synthesis of nitrogen-doped graphene via pyrolysis of graphene oxide and urea, and its electrocatalytic activity toward the oxygen-reduction reaction, Adv. Energy Mater., 2012, 2(7), 884-888.

14 Y. Li, Y. Zhao, H. Cheng, Y. Hu, G. Shi, L. Dai and L. Qu, Nitrogen-doped graphene quantum dots with oxygen-rich functional groups, J. Am. Chem. Soc., 2011, 134(1), 15-18.

15 K. Kwon, Y. J. Sa, J. Y. Cheon and S. H. Joo, Ordered mesoporous carbon nitrides with graphitic frameworks as metal-free, highly durable, methanol-tolerant oxygen reduction catalysts in an acidic medium, Langmuir, 2011, 28(1), 991-996.

16 X. Qiao, S. Liao, C. You and R. Chen, Phosphorus and nitrogen dual doped and simultaneously reduced graphene oxide with high surface area as efficient metal-free electrocatalyst for oxygen reduction, Catalysts, 2015, 5(2), 981-991.

17 Z.-J. Lu, S.-J. Bao, Y.-T. Gou, C.-J. Cai, C.-C. Ji, M.-W. Xu, J. Song and R. Wang, Nitrogen-doped reduced-graphene oxide as an efficient metal-free electrocatalyst for oxygen reduction in fuel cells, RSC Adv., 2013, 3(12), 3990-3995.

18 C. Uthaisar and V. Barone, Edge effects on the characteristics of Li diffusion in graphene, Nano Lett., 2010, 10(8), 28382842.

19 V. Georgakilas, J. A. Perman, J. Tucek and R. Zboril, Broad family of carbon nanoallotropes: classification, chemistry, and applications of fullerenes, carbon dots, nanotubes, graphene, nanodiamonds, and combined superstructures, Chem. Rev., 2015, 115(11), 4744-4822.

20 A. C. Ferrari, F. Bonaccorso, V. Fal'Ko, K. S. Novoselov, S. Roche, P. Bøggild, S. Borini, F. H. L. Koppens, V. Palermo and N. Pugno, Science and technology 
roadmap for graphene, related two-dimensional crystals, and hybrid systems, Nanoscale, 2015, 7(11), 4598-4810.

21 A. Narita, X.-Y. Wang, X. Feng and K. Müllen, New advances in nanographene chemistry, Chem. Soc. Rev., 2015, 44(18), 6616-6643.

22 T. H. Han, Y.-K. Huang, A. T. L. Tan, V. P. Dravid and J. Huang, Steam etched porous graphene oxide network for chemical sensing, J. Am. Chem. Soc., 2011, 133(39), 1526415267.

23 X. Wang, L. Jiao, K. Sheng, C. Li, L. Dai and G. Shi, Solutionprocessable graphene nanomeshes with controlled pore structures, Sci. Rep., 2013, 3, 1996.

24 O. Akhavan, Graphene nanomesh by $\mathrm{ZnO}$ nanorod photocatalysts, ACS Nano, 2010, 4(7), 4174-4180.

25 N. S. Safron, M. Kim, P. Gopalan and M. S. Arnold, BarrierGuided Growth of Micro-and Nano-Structured Graphene, Adv. Mater., 2012, 24(8), 1041-1045.

26 M. D. Fischbein and M. Drndić, Electron beam nanosculpting of suspended graphene sheets, Appl. Phys. Lett., 2008, 93(11), 113107.

27 J. Huang, J. Wang, C. Wang, H. Zhang, C. Lu and J. Wang, Hierarchical porous graphene carbon-based supercapacitors, Chem. Mater., 2015, 27(6), 2107-2113.

28 Z. Lin, G. H. Waller, Y. Liu, M. Liu and C.-P. Wong, Simple preparation of nanoporous few-layer nitrogen-doped graphene for use as an efficient electrocatalyst for oxygen reduction and oxygen evolution reactions, Carbon, 2013, 53, 130-136.

29 A. Rashidi, L. Mahmudian and H. Dehghani, Producing graphene and nanoporous graphene, US 20160060123 A1, 2015.

30 X. Li, H. Wang, J. T. Robinson, H. Sanchez, G. Diankov and H. Dai, Simultaneous nitrogen doping and reduction of graphene oxide, J. Am. Chem. Soc., 2009, 131(43), 1593915944.

31 Y. Su, Y. Zhang, X. Zhuang, S. Li, D. Wu, F. Zhang and $\mathrm{X}$. Feng, Low-temperature synthesis of nitrogen/sulfur codoped three-dimensional graphene frameworks as efficient metal-free electrocatalyst for oxygen reduction reaction, Carbon, 2013, 62, 296-301.

32 Y. Liu, K. Li, Y. Liu, L. Pu, Z. Chen and S. Deng, The highperformance and mechanism of P-doped activated carbon as a catalyst for air-cathode microbial fuel cells, J. Mater. Chem. A, 2015, 3(42), 21149-21158.

33 Z.-J. Lu, M.-W. Xu, S.-J. Bao, K. Tan, H. Chai, C.-J. Cai, C.-C. Ji and Q. Zhang, Facile preparation of nitrogen-doped reduced graphene oxide as a metal-free catalyst for oxygen reduction reaction, J. Mater. Sci., 2013, 48(23), 8101-8107.

34 H. M. Ju, S. H. Choi and S. H. Huh, X-ray diffraction patterns of thermally-reduced graphenes, J. Korean Phys. Soc., 2010, 57(61), 1649-1652.

35 Z. Xing, Z. Ju, Y. Zhao, J. Wan, Y. Zhu, Y. Qiang and Y. Qian, One-pot hydrothermal synthesis of Nitrogen-doped graphene as high-performance anode materials for lithium ion batteries, Sci. Rep., 2016, 6, 26146.

36 W. Lv, D.-M. Tang, Y.-B. He, C.-H. You, Z.-Q. Shi, X.-C. Chen, C.-M. Chen, P.-X. Hou, C. Liu and Q.-H. Yang, Lowtemperature exfoliated graphenes: vacuum-promoted exfoliation and electrochemical energy storage, ACS Nano, 2009, 3(11), 3730-3736.

37 A. C. Ferrari, J. C. Meyer, V. Scardaci, C. Casiraghi, M. Lazzeri, F. Mauri, S. Piscanec, D. Jiang, K. S. Novoselov and S. Roth, Raman spectrum of graphene and graphene layers, Phys. Rev. Lett., 2006, 97(18), 187401.

38 X. Li, Y. Fang, S. Zhao, J. Wu, F. Li, M. Tian, X. Long, J. Jin and J. Ma, Nitrogen-doped mesoporous carbon nanosheet/ carbon nanotube hybrids as metal-free bi-functional electrocatalysts for water oxidation and oxygen reduction, J. Mater. Chem. A, 2016, 4(34), 13133-13141.

39 Z. Lin, G. H. Waller, Y. Liu, M. Liu and C.-P. Wong, 3D Nitrogen-doped graphene prepared by pyrolysis of graphene oxide with polypyrrole for electrocatalysis of oxygen reduction reaction, Nano Energy, 2013, 2(2), 241-248.

40 L. Lai, J. R. Potts, D. Zhan, L. Wang, C. K. Poh, C. Tang, H. Gong, Z. Shen, J. Lin and R. S. Ruoff, Exploration of the active center structure of nitrogen-doped graphene-based catalysts for oxygen reduction reaction, Energy Environ. Sci., 2012, 5(7), 7936-7942.

41 D. Guo, R. Shibuya, C. Akiba, S. Saji, T. Kondo and J. Nakamura, Active sites of nitrogen-doped carbon materials for oxygen reduction reaction clarified using model catalysts, Science, 2016, 351(6271), 361-365.

42 B. Li, X. Y. Sun and D. Su, Calibration of the basic strength of the nitrogen groups on the nanostructured carbon materials, Phys. Chem. Chem. Phys., 2015, 17(10), 6691-6694.

43 J. Zhang and L. Dai, Heteroatom-Doped Graphitic Carbon Catalysts for Efficient Electrocatalysis of Oxygen Reduction Reaction, ACS Catal., 2015, 5(12), 7244-7253.

44 R. Silva, D. Voiry, M. Chhowalla and T. Asefa, Efficient MetalFree Electrocatalysts for Oxygen Reduction: PolyanilineDerived N- and O-Doped Mesoporous Carbons, J. Am. Chem. Soc., 2013, 135(21), 7823-7826.

45 W. Yang, T.-P. Fellinger and M. Antonietti, Efficient MetalFree Oxygen Reduction in Alkaline Medium on HighSurface-Area Mesoporous Nitrogen-Doped Carbons Made from Ionic Liquids and Nucleobases, J. Am. Chem. Soc., 2011, 133(2), 206-209.

46 X. Zhou, Z. Yang, H. Nie, Z. Yao, L. Zhang and S. Huang, Catalyst-free growth of large scale nitrogen-doped carbon spheres as efficient electrocatalysts for oxygen reduction in alkaline medium, J. Power Sources, 2011, 196(23), 9970-9974.

47 C. Han, J. Wang, Y. Gong, X. Xu, H. Li and Y. Wang, Nitrogen-doped hollow carbon hemispheres as efficient metal-free electrocatalysts for oxygen reduction reaction in alkaline medium, J. Mater. Chem. A, 2014, 2(3), 605-609.

48 D.-S. Yang, D. Bhattacharjya, S. Inamdar, J. Park and J.-S. Yu, Phosphorus-Doped Ordered Mesoporous Carbons with Different Lengths as Efficient Metal-Free Electrocatalysts for Oxygen Reduction Reaction in Alkaline Media, J. Am. Chem. Soc., 2012, 134(39), 16127-16130.

49 Y. Li, Y. Zhao, H. Cheng, Y. Hu, G. Shi, L. Dai and L. Qu, Nitrogen-Doped Graphene Quantum Dots with OxygenRich Functional Groups, J. Am. Chem. Soc., 2012, 134(1), 15-18. 
50 Q. Li, S. Zhang, L. Dai and L.-S. Li, Nitrogen-Doped Colloidal Graphene Quantum Dots and Their Size-Dependent Electrocatalytic Activity for the Oxygen Reduction Reaction, J. Am. Chem. Soc., 2012, 134(46), 18932-18935.

51 X. Sun, Y. Zhang, P. Song, J. Pan, L. Zhuang, W. Xu and W. Xing, Fluorine-Doped Carbon Blacks: Highly Efficient Metal-Free Electrocatalysts for Oxygen Reduction Reaction, ACS Catal., 2013, 3(8), 1726-1729.

52 Y. Zhan, J. Huang, Z. Lin, X. Yu, D. Zeng, X. Zhang, F. Xie, W. Zhang, J. Chen and H. Meng, Iodine/nitrogen co-doped graphene as metal free catalyst for oxygen reduction reaction, Carbon, 2015, 95, 930-939.

53 Z. Liu, H. Nie, Z. Yang, J. Zhang, Z. Jin, Y. Lu, Z. Xiao and S. Huang, Sulfur-nitrogen co-doped three-dimensional carbon foams with hierarchical pore structures as efficient metal-free electrocatalysts for oxygen reduction reactions, Nanoscale, 2013, 5(8), 3283-3288.

54 S. Yang, X. Feng, X. Wang and K. Müllen, Graphene-Based Carbon Nitride Nanosheets as Efficient Metal-Free Electrocatalysts for Oxygen Reduction Reactions, Angew. Chem., Int. Ed., 2011, 50(23), 5339-5343.

55 J. Chen, X. Wang, X. Cui, G. Yang and W. Zheng, Amorphous carbon enriched with pyridinic nitrogen as an efficient metal-free electrocatalyst for oxygen reduction reaction, Chem. Commun., 2014, 50(5), 557-559.

56 R. Gokhale, S. M. Unni, D. Puthusseri, S. Kurungot and S. Ogale, Synthesis of an efficient heteroatom-doped carbon electro-catalyst for oxygen reduction reaction by pyrolysis of protein-rich pulse flour cooked with $\mathrm{SiO} 2$ nanoparticles, Phys. Chem. Chem. Phys., 2014, 16(9), 42514259.

57 F. Pan, J. Jin, X. Fu, Q. Liu and J. Zhang, Advanced Oxygen Reduction Electrocatalyst Based on Nitrogen-Doped Graphene Derived from Edible Sugar and Urea, ACS Appl. Mater. Interfaces, 2013, 5(21), 11108-11114.

58 W. Xia, J. Masa, M. Bron, W. Schuhmann and M. Muhler, Highly active metal-free nitrogen-containing carbon catalysts for oxygen reduction synthesized by thermal treatment of polypyridine-carbon black mixtures, Electrochem. Commun., 2011, 13(6), 593-596.

$59 \mathrm{X}$. Bo and L. Guo, Ordered mesoporous boron-doped carbons as metal-free electrocatalysts for the oxygen reduction reaction in alkaline solution, Phys. Chem. Chem. Phys., 2013, 15(7), 2459-2465.

60 N. Brun, S. A. Wohlgemuth, P. Osiceanu and M. M. Titirici, Original design of nitrogen-doped carbon aerogels from sustainable precursors: application as metal-free oxygen reduction catalysts, Green Chem., 2013, 15(9), 2514-2524.

61 H. Wang, X. Bo, C. Luhana and L. Guo, Nitrogen doped large mesoporous carbon for oxygen reduction electrocatalyst using DNA as carbon and nitrogen precursor, Electrochem. Commun., 2012, 21, 5-8.

62 J. Park, Y. Nabae, T. Hayakawa and M.-A. Kakimoto, Highly Selective Two-Electron Oxygen Reduction Catalyzed by Mesoporous Nitrogen-Doped Carbon, ACS Catal., 2014, 4(10), 3749-3754.

63 A. Muthukrishnan, Y. Nabae, T. Okajima and T. Ohsaka, Kinetic Approach to Investigate the Mechanistic Pathways of Oxygen Reduction Reaction on Fe-Containing N-Doped Carbon Catalysts, ACS Catal., 2015, 5(9), 5194-5202.

64 X. Wang, J.-S. Lee, Q. Zhu, J. Liu, Y. Wang and S. Dai, Ammonia-Treated Ordered Mesoporous Carbons as Catalytic Materials for Oxygen Reduction Reaction, Chem. Mater., 2010, 22(7), 2178-2180.

65 D. Yu, Q. Zhang and L. Dai, Highly Efficient Metal-Free Growth of Nitrogen-Doped Single-Walled Carbon Nanotubes on Plasma-Etched Substrates for Oxygen Reduction, J. Am. Chem. Soc., 2010, 132(43), 15127-15129.

66 Y. Wang, M. Zhu, Y. Li, M. Zhang, X. Xue, Y. Shi, B. Dai, $\mathrm{X}$. Guo and F. Yu, Heteroatom-doped porous carbon from methyl orange dye wastewater for oxygen reduction, Green Energy Environ., 2017, in press.

67 B. Men, Y. Sun, M. Li, C. Hu, M. Zhang, L. Wang, Y. Tang, Y. Chen, P. Wan and J. Pan, Hierarchical Metal-Free Nitrogen-Doped Porous Graphene/Carbon Composites as an Efficient Oxygen Reduction Reaction Catalyst, ACS Appl. Mater. Interfaces, 2016, 8(2), 1415-1423. 